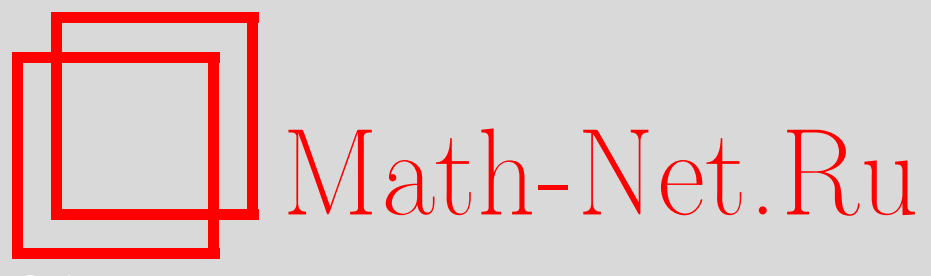

А. А. Борисенко, О цилиндричности подмногообразий, содержащих прямую, в пространстве Минковского, $\mathrm{Ma}$ тем. сб., 2014, том 205, номер 7, 25-42

DOI: https://doi.org/10.4213/sm8327

Использование Общероссийского математического портала Math-Net.Ru подразумевает, что вы прочитали и согласны с пользовательским соглашением http://www.mathnet.ru/rus/agreement

Параметры загрузки:

IP : 54.224 .60 .19

26 апреля 2023 г., 14:22:41

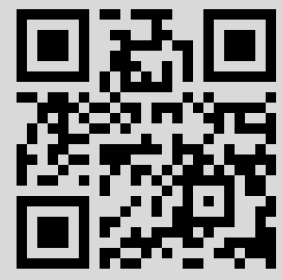




\section{А. А. Борисенко \\ О цилиндричности подмногообразий, содержащих прямую, в пространстве Минковского}

Рассматриваются финслеровы подмногообразия в пространстве Минковского и, в частности, в пространстве Рандерса. Дано обобщение теорем Топоногова, Чигера-Громолла на случай пространств Рандерса. Доказаны достаточные условия цилиндричности полных финслеровых подмногообразий в пространстве Минковского. Найдены условия, когда из выпуклости гиперповерхности в пространстве Рандерса следует положительность флаговой кривизны.

Библиография: 17 названий.

Ключевые слова: флаговая кривизна, кривизна Риччи, финслерова метрика, риманова метрика, внешний нуль-индекс, распределение.

DOI: $10.4213 / \mathrm{sm} 8327$

\section{§1. Введение}

Если полное двумерное риманово многообразие неотрицательной гауссовой кривизны содержит прямую (полную геодезическую, которая является кратчайшей на каждом отрезке), то многообразие является плоским. Это было доказано С. Кон-Фоссеном в [1]. В. А. Топоногов обобщил этот результат на многомерный случай. Он доказал, что если на полном некомпактном римановом многообразии $M^{n}$ неотрицательной секционной кривизны существует $k$ линейно независимых прямых, то $M^{n}$ есть риманово произведение $M^{n}=M^{n-k} \times E^{k}$, где $E^{k}$ изометрично евклидову пространству, $M^{n-k}$ - полное риманово многообразие неотрицательной секционной кривизны (см. [2]).

На случай неотрицательной кривизны Риччи теорема была обобщена Дж. Чичером и Д. Громоллом в [3]. В псевдоримановом случае теорема была доказана Дж. Эшенбургом и Г. Галловеем в [4] и [5]. С. Охта доказал аналогичные теоремы о расщеплении на случай финслеровых пространств Бервальда с неотрицательной взвешенной кривизной Риччи в [6].

С другой стороны, для полных подмногообразий в евклидовом пространстве были найдены условия, когда подмногообразия являлись цилиндрами. Пусть $M^{l}$ - полное регулярное класса $C^{3}$ подмногообразие в евклидовом пространстве $E^{n}$. Если во всех точках подмногообразия нуль-индекс $\mu(Q)$ больше или равен $k$ и кривизна Риччи неотрицательна, то подмногообразие $M^{l}$ является цилиндром с $k$-мерной образующей евклидовой плоскостью $E^{k}$ (см. [7]). При условии неотрицательности секционной кривизны теорема была доказана $\Phi$. Хартманом в [8], для полных гиперповерхностей нулевой секционной кривизны - Ф. Хартманом и Л. Ниренбергом в [9] (так как в этом случае $\mu \geqslant l-1$ ), 
для гиперповерхностей неотрицательной секционной кривизны - Р. Сакстедером в [10].

Без ограничений на внутреннюю геометрии подмногообразия была доказана следующая

Теорема 1 (см. [7]). Пусть $M^{l}$ - полное регулярное связное подмногообразие в евклидовом пространстве $E^{n}$. Если:

1) нуль-индекс $\mu(Q)$ равен $k$ во всех точках подмногообразия,

2) тип точки $j(Q)$ равен 0 для всех точек подмногообразия, то подмногообразие $M^{l}$ есть иилиндр с евклидовой $k$-мерной образующей $E^{k}$.

В настоящей работе находятся условия, при которых полное финслерово подмногообразие $M^{l}$ неотрицательной кривизны Риччи в пространстве Минковского и в частном случае в пространстве Рандерса $R^{n}$ является цилиндром, если на нем лежит прямая объемлющего пространства или нуль-индекс $\mu$ больше 0 в каждой точке.

Условимся, что индексы принимают такие значения: $i, j, k=1, \ldots, l$; $\alpha, \beta, \gamma=1, \ldots, n-l+1 ; a, b, c=1, \ldots, n+1$.

Пространством Минковского $M^{n+1}$ (см. [11], [12]) называется пара $\left(V^{n+1}, S\right)$, где $V^{n+1}$ - это $(n+1)$-мерное вещественное пространство с декартовыми координатами $y^{1}, \ldots, y^{n+1}$.

Норма Минковского $S: V^{n+1} \rightarrow[0, \infty)$ обладает свойствами:

1) $S \in C^{\infty}\left(V^{n+1} \backslash\{0\}\right)$;

2) для любого положительного числа $q S(q y)=q S(y)$;

3) симметричная билинейная форма

$$
g_{a b}=\frac{1}{2} \frac{\partial^{2} S^{2}}{\partial y^{a} \partial y^{b}}
$$

положительно определена на $V^{n+1}$.

Метрика $S$, индуцируемая на поверхности $M^{l} \subset M^{n+1}$, является финслеровой, т.е. $S(x ; y): T_{P} M^{l} \rightarrow[0,+\infty)$ и для произвольной точки $\left.P \in M^{l} S\right|_{T_{P} M^{l}}-$ норма Минковского на $T_{P} M^{l}$.

Пространством Рандерса $R^{n+1}$ будем называть пространство Минковского с нормой $S$ вида

$$
S(y)=|y|_{E}+\beta(y),
$$

где $|y|_{E}=\sqrt{\sum_{i=1}^{n+1}\left(y^{a}\right)^{2}}-$ евклидова норма, $\beta(y)=b_{a} y^{a}-$ линейная форма.

Необходимым и достаточным условием того, что данная функция (1.1) является нормой Минковского, является условие на евклидову длину вектора $b=\left(b_{1}, \ldots, b_{n+1}\right)|b|<1$. Условимся считать пространство неевклидовым, т.е. $|b| \neq 0$.

Будем рассматривать $C^{3}$-гладкое подмногообразие $M^{l}$ как множество точек в двух различных пространствах: в пространстве Рандерса (обозначая поверхность в таком случае $M^{l} \subset R^{n}$ с индуцированной финслеровой метрикой) и в наложенном евклидовом пространстве (в этом случае обозначая поверхность $\bar{M}^{l} \subset E^{n}$ с индуцированной римановой метрикой). 
Аналогом понятия секционной кривизны римановых пространств является флаговая кривизна финслеровых пространств (см. [11]), которая может быть определена как секционная кривизна римановой метрики

$$
g=\sqrt{g_{i j}(x, Z) u^{i} u^{j}}
$$

для некоторого геодезического поля $Z$ финслерового пространства, где

$$
g_{i j}=\frac{1}{2} \frac{\partial^{2} S^{2}(x, y)}{\partial y^{i} \partial y^{j}}
$$

- коэффициенты метрического тензора финслерового пространства, задаваемого функцией $S(x, y)$.

\section{§ 2. Формулировка теорем}

Подмногообразие в пространстве Минковского $M^{n+1}$ называется иилиндром с образующей вполне геодезической плоскостью $M^{k}$, если через каждую точку подмногообразия проходит плоскость, параллельная $M^{k}$, которая принадлежит подмногообразию.

Для полных подмногообразий в пространстве Минковского справедлива

Теорема 2. Пусть $M^{l}$ - полное регулярное связное подмногообразие в пространстве Минковского $M^{n}$. Если:

1) нуль-индекс $\mu(Q)$ равен $k$ во всех точках подмногообразия,

2) тип точки $j(Q)$ равен 0 для всех точек подмногообразия, то подмногообразие $M^{l}$ есть иилиндр с $k$-мерной образующей.

Для гиперповерхностей в пространстве Рандерса имеет место

Теорема 3. Пусть $M^{l}$ - полная гиперповерхность неотрицательной кривизны Риччи в пространстве Рандерса $R^{l+1}$. Если на $M^{l}$ лежит прямая пространства Рандерса, то $M^{l}$ является иилиндром.

Теорема 4. Пусть $M^{l}$ - полная гиперповерхность неотрицателъной кривизны Риччи в пространстве Рандерса $R^{l+1}$. Если нуль-индекс $\mu$ больше или равен $k$, то $M^{l}$ является иилиндром с образующей $k$-мерной вполне геодезической плоскостью $R^{k}$.

Теорема 5. Пусть $M^{l}$ - полная гиперповерхность неотрицательной кривизны Риччи в пространстве Рандерса. Если нуль-индекс Риччи:

1) $\nu_{0}=l$, то $M^{l}$ является иилиндром $c(l-1)$-мерной образующей;

2) $l>\nu_{0} \geqslant k$, то $M^{l}$ является иилиндром с $k$-мерной образующей.

Пусть $k(u)$ - вектор нормальной кривизны подмногообразия $\bar{M}^{l}$ в евклидовом наложенном пространстве $E^{n}$.

Теорема 6. Пусть $M^{l}$ - полное подмногообразие в пространстве Рандерса $R^{n}$. Если кривизна Риччи $M^{l}$ удовлетворяет неравенству

$$
\operatorname{Ric}(u) \geqslant \frac{3}{4}(l-1)\langle k(u), b\rangle^{2}
$$


и на подмногообразии лежит прямая обгемлющего пространства, то подмногообразие является иилиндром.

Теорема 7. Пусть $M^{l}$ - полное подмногообразие в пространстве Рандерса $R^{n}$, внешний нуль-индекс которого в каждой точке есть $\mu \geqslant \mu_{0}>0$. Если финслерова кривизна Риччи

$$
\operatorname{Ric}(u) \geqslant \frac{3}{4}(l-1)\langle k(u), b\rangle^{2},
$$

то $M^{l}$ является иилиндром с $\mu_{0}$-мерной вполне геодезической плоской образующей.

\section{§ 3. Доказательство лемм}

Лемма 1. Метрика, индуиируемая на подмногообразиях $M^{l}$ пространства Рандерса $R^{n+1}$ метрикой $S=\alpha+\beta$, проективно эквивалентна римановой метрике на $\bar{M}^{l}$, индуиируемой евклидовой метрикой, заданной в наложенном евклидовом пространстве $E^{n+1}$.

ДокАЗАТЕЛьСтво. Зададим подмногообразие $M^{l}$ в окрестности некоторой точки $P$ явным образом:

$$
x^{l+\alpha}=f^{\alpha}\left(x^{1}, \ldots, x^{l}\right) .
$$

Индуцируемая финслерова метрика $S(x, u)$ на $M^{l}$ для вектора

$$
Y=\left(u^{1}, \ldots, u^{l}, u^{i} f_{i}^{l+1}, \ldots, u^{i} f_{i}^{n+1-l}\right) \in T_{P} M^{l}
$$

имеет вид

$$
S(x, u)=\sqrt{\left(\delta_{i j}+\sum_{\alpha=1}^{n+1-l} f_{i}^{l+\alpha} f_{j}^{l+\alpha}\right) u^{i} u^{j}}+\left(b_{i}+b_{l+\gamma} f_{i}^{\gamma}\right) u^{i}=\alpha+\gamma .
$$

Необходимым и достаточным условием того, что метрика $F$ проективно эквивалентна римановой метрике

$$
\alpha(u)=\sqrt{\left(\delta_{i j}+\sum_{\alpha=1}^{n+1-l} f_{i}^{l+\alpha} f_{j}^{l+\alpha}\right), u^{i} u^{j}}
$$

является условие замкнутости формы $\gamma(u)=c_{i}(x) u^{i}$, где $c_{i}(x)=b_{i}+b_{l+\alpha} f_{i}^{\alpha}$, т.e.

$$
\frac{\partial c_{i}(x)}{\partial x^{j}}=\frac{\partial c_{j}(x)}{\partial x^{i}}
$$

Действительно,

$$
\begin{aligned}
\frac{\partial c_{i}(x)}{\partial x^{j}} & =\frac{\partial b_{i}}{\partial x^{j}}+\frac{\partial b_{l+\alpha}}{\partial x^{j}} f_{i}^{\alpha}+b_{l+\alpha} \frac{\partial f^{\alpha}}{\partial x^{i} \partial x^{j}}, \\
\frac{\partial c_{j}(x)}{\partial x^{i}} & =\frac{\partial b_{j}}{\partial x^{i}}+\frac{\partial b_{l+\alpha}}{\partial x^{i}} f_{j}^{\alpha}+b_{l+\alpha} \frac{\partial f^{\alpha}}{\partial x^{i} \partial x^{j}} .
\end{aligned}
$$

Так как $b_{a}-$ константы, то условия замкнутости выполняются. 
Лемма 2. Пусть $M^{l}$ - подмногообразие в пространстве Рандерса $R^{n+1}$, $\bar{M}^{l}$ - подмногообразие в наложенном евклидовом пространстве $E^{n+1}$, которое как множество точек совпадает с подмногообразием $M^{l}$. Тогда флаговая кривизна $K_{\sigma}(u)$ подмногообразия $M^{l}$ и секционная кривизна $\bar{K}_{\sigma}$ подмногообразия $\bar{M}^{l}$ связаны равенством

$$
K_{\sigma}(u)=\frac{\bar{K}_{\sigma}+\frac{3}{4 S^{2}}\langle k(u), b\rangle^{2}-\frac{1}{2 S} \frac{\partial}{\partial s}\langle k(u), b\rangle}{S^{2}} .
$$

ДокАзАТЕЛЬство. Флаговая кривизна финслеровой метрики $S(x, y)$ определяется следующим образом:

$$
K(\sigma, u)=\frac{g_{u}\left(R_{u}(v), v\right)}{g_{u}(u, u) g_{u}(v, v)-g_{u}^{2}(v, u)},
$$

где $\sigma=\operatorname{span}\{v, u\}, R_{u}(v)=R_{k}^{i}(u) v^{k}, R_{k}^{i}(u)=u^{i} R_{j k l}^{i}(v) u^{l}$, а $R_{j k l}^{i}$ - тензор кривизны финслеровой метрики $S(x, u)$ на $M^{l}$ (см. [12; п. 2.2, (2.45)]).

Для проективно эквивалентных метрик Рандерса и Римана верно следующее соотношение (см. [11; п. 6.1, (6.10)]):

$$
R_{k}^{i}=\bar{R}_{k}^{i}+\left(\frac{3 \Phi^{2}}{4 S^{2}}-\frac{\Psi}{2 S}\right)\left(\delta_{k}^{i}-\frac{S_{u^{k}}}{S} u^{i}\right)+\tau_{k} u^{i},
$$

где $R_{k}^{i}, \bar{R}_{k}^{i}$ - соответственно тензоры кривизны метрики Рандерса и соответствующей римановой метрики,

$$
\Phi=c_{i, j}(x) u^{i} u^{j}, \quad \Psi=c_{i, j k} u^{i} u^{j} u^{k}, \quad \tau_{k}=\frac{1}{S(u)}\left(c_{i, j k}-c_{i, k j}\right) u^{i} u^{j},
$$

$c_{i, j}$ - ковариантная производная ковектора $c_{i}$ в римановой связности метрики $\alpha$.

Метрический тензор римановой метрики $\alpha$ имеет вид

$$
\bar{g}_{i j}(x)=\delta_{i j}+\left\langle F_{i}, F_{j}\right\rangle,
$$

где $F$ есть вектор с компонентами $f^{\alpha}(x), F_{i}=\partial F / \partial x^{i},\langle\cdot, \cdot\rangle$ - скалярное произведение в евклидовом пространстве в ортонормированном базисе. Введем наложенное евклидово пространство $E^{n+1}$, которое как множество точек совпадает с пространством Рандерса, а подмногообразие $\bar{M}^{l} \subset E^{n+1}$ как множество точек совпадает с подмногообразием $M^{l} \subset R^{n+1}$. На подмногообразии $\bar{M}^{l}$ индуцируется риманова метрика $\alpha$. Возьмем точку $P \in \bar{M}^{l}$ за начало ортогональной декартовой системы координат, касательное пространство $T_{P} \bar{M} l$ за пространство переменных $x^{1}, \ldots, x^{l}$; нормальное пространство $N_{P} \bar{M}^{l}$ - это пространство переменных $x^{l+\alpha}$. Эти координаты выбраны и в пространстве Рандерса. В этих координатах $\bar{M}^{l}$ задается в виде (3.1) и в точке $P$

$$
\frac{\partial f^{\alpha}}{\partial x^{i}}(0)=0
$$

Тогда из (3.3) и (3.7) мы получим

$$
\begin{gathered}
\frac{\partial \bar{g}_{i j}}{\partial x^{k}}(0)=0 \\
\bar{\Gamma}_{i j}^{k}(0)=0 .
\end{gathered}
$$


Найдем ковариантные производные:

$$
\begin{gathered}
c_{i, j}(0)=\left(b_{i}+b_{l+\alpha} f_{i}^{\alpha}\right)_{j}=b_{l+\alpha} f_{i j}^{\alpha}, \\
c_{i, j k}=\frac{\partial c_{i, j}}{\partial x^{k}}=\frac{\partial c_{i}}{\partial x^{j} \partial x^{k}}-\frac{\partial \bar{\Gamma}_{i j}^{s}}{\partial x^{k}} b_{s}, \\
\frac{\partial \bar{\Gamma}_{i j}^{s}}{\partial x^{k}}=\frac{\partial}{\partial x^{k}}\left(\frac{\bar{g}^{s m}}{2} \cdot\left(\frac{\partial \bar{g}_{i m}}{\partial x^{j}}+\frac{\partial \bar{g}_{j m}}{\partial x^{i}}-\frac{\partial \bar{g}_{i j}}{\partial x^{m}}\right)\right) .
\end{gathered}
$$

Из (3.8), (3.12) следует, что

$$
\frac{\partial \bar{\Gamma}_{i j}^{s}}{\partial x^{k}}=\frac{1}{2}\left(\frac{\partial \bar{g}_{i s}}{\partial x^{j} \partial x^{k}}+\frac{\partial \bar{g}_{j s}}{\partial x^{i} \partial x^{k}}-\frac{\partial \bar{g}_{i j}}{\partial x^{s} \partial x^{k}}\right) .
$$

Из (3.7), (3.13) получаем

$$
\frac{\partial \bar{g}_{i j}}{\partial x^{k}}=\left\langle F_{i k}, F_{j}\right\rangle+\left\langle F_{i}, F_{j k}\right\rangle
$$

Так как в точке $P$ вектор $F_{i}$ равен 0 , то

$$
\frac{\partial \bar{g}_{i j}}{\partial x^{s} \partial x^{k}}(0)=\left\langle F_{i k}, F_{j s}\right\rangle+\left\langle F_{i s}, F_{j k}\right\rangle .
$$

Подставив (3.15) в (3.13), мы получим

$$
\frac{\partial \bar{\Gamma}_{i j}^{s}}{\partial x^{k}}=\left\langle F_{i j}, F_{s k}\right\rangle
$$

Подставим (3.10) и (3.15) в (3.11). Тогда

$$
c_{i, j k}(0)=b_{l+\alpha} f_{i j k}^{\alpha}-\sum_{s}\left\langle F_{i j}, F_{s k}\right\rangle b_{s}=b_{l+\alpha} f_{i j k}^{\alpha}-\left\langle F_{i j}, \sum_{s} F_{s k} b_{s}\right\rangle .
$$

Рассмотрим произвольную нормальную геодезическую, проходящую через точку $P$ на подмногообразия $\bar{M}^{l}$ в $E^{n+1}$. Ее радиус-вектор $r(s): x^{i}=x^{i}(s)$, $x^{l+\alpha}=f^{\alpha}\left(x^{1}(s), \ldots, x^{l}(s)\right), s-$ длина дуги. Ее единичная главная нормаль $\nu$ в точке $P$ совпадает с нормалью $\nu_{1}$ к подмногообразию $\bar{M}^{l}$ и имеет координаты $(\underbrace{0, \ldots, 0}_{l}, 1,0, \ldots, 0)$, вектор кривизны геодезической

$$
r^{\prime \prime}(s)=k(u)=\sum_{\alpha=1}^{n-l+1} k^{\alpha} \nu_{\alpha}=k \nu
$$

где $\nu_{\alpha}$ - ортогональный базис нормалей, ковариантные производные которых в нормальной связности равны нулю в точке $P, k^{\alpha}$ - нормальные кривизны подмногообразия относительно нормалей $\nu_{\alpha}$ в направлении касательного вектора $u$ геодезической. Вектор $k(u)$ является также вектором нормальной кривизны подмногообразия $\bar{M}^{l}$ в направлении касательного вектора $u$.

Так как в точке $P$

$$
f_{i}^{\alpha}(P)=0, \quad \frac{\partial^{2} x^{i}}{\partial s^{2}}(P)=0,
$$


то в точке $P$

$$
f_{s s s}^{\alpha}=f_{i j k}^{\alpha} \frac{\partial x^{i}}{\partial s} \frac{\partial x^{j}}{\partial s} \frac{\partial x^{k}}{\partial s}=f_{i j k}^{\alpha} u^{i} u^{j} u^{k},
$$

где $u=\left(u^{1}, \ldots, u^{l}\right)$ - координаты касательного вектора к геодезической.

Из (3.3), (3.10) следует, что в точке $P$

$$
\Phi(P)=b_{l+\alpha} f_{i j}^{\alpha} u^{i} u^{j}=\langle b, k(u)\rangle .
$$

Из (3.6), (3.17), (3.18) следует, что

$$
\Psi(P)=b_{l+\alpha} f_{i j k}^{\alpha} u^{i} u^{j} u^{k}-\left\langle F_{i j}, \sum_{s} b_{s} F_{s k}\right\rangle u^{i} u^{j} u^{k} .
$$

Выберем базис единичных нормалей $n_{\alpha}$ к подмногообразию $\bar{M}^{l}$,

$$
n_{\alpha}=\frac{1}{\sqrt{1+\left(\operatorname{grad} f^{\alpha}\right)^{2}}}\left(f_{1}^{\alpha}, \ldots, f_{l}^{\alpha}, 0, \ldots,-1, \ldots, 0\right),
$$

где -1 стоит на месте с номером $l+\alpha$. Стандартным образом ортогонализуем этот базис, чтобы он был ортогональным в окрестности точки $P$, взяв векторы $\nu_{1}=n_{1}, \nu_{2}=\operatorname{span}\left(n_{1}, n_{2}\right)$ и т.д. Легко подсчитать, что ковариантные производные $\nu_{\alpha}$ в нормальной связности в точке $P$ равны нулю, $\nabla_{e_{s}}^{\perp} \nu_{\alpha}=0$, где $e_{s}-$ ортогональный базис касательного пространства $T_{P} \bar{M}^{l}$. Поэтому

$$
\left.\frac{\partial k^{\alpha} \nu_{\alpha}}{\partial s}\right|_{P}=\frac{\partial k^{\alpha}}{\partial u^{i}} \frac{\partial x^{i}}{\partial s} \nu_{\left.\alpha\right|_{P}}-k^{\alpha} \sum_{i} f_{i k}^{\alpha} \frac{\partial x^{k}}{\partial s} e_{i} .
$$

Так как

$$
\left.k^{\alpha}\right|_{P}=f_{i j}^{\alpha} \frac{\partial x^{i}}{\partial s} \frac{\partial x^{j}}{\partial s},\left.\quad \frac{\partial k^{\alpha}}{\partial s}\right|_{P}=f_{i j k}^{\alpha} \frac{\partial x^{i}}{\partial s} \frac{\partial x^{j}}{\partial s} \frac{\partial x^{k}}{\partial s}, \quad u^{i}=\frac{\partial x^{i}}{\partial s},
$$

то из предыдущей формулы и выражения для $\Psi(P)$ получается

$$
\Psi(P)=\frac{\partial}{\partial s}\langle k(u), b\rangle .
$$

Из (3.6), (3.16) мы получим

$$
\tau_{k}=\frac{1}{S}\left(-\left\langle k(u), \sum_{s=1}^{l} F_{s k} b_{s}\right\rangle+\left\langle F_{i k} u^{i}, \sum_{s=1}^{l} b_{s} F_{s j} u^{j}\right\rangle\right) .
$$

Найдем связь между $R_{k}^{i}, \bar{R}_{k}^{i}$.

При условии $|u|_{E}=1$ из (3.2) следует, что

$$
S_{u^{k}}(P)=b_{k}+u^{k} .
$$

Из (3.5), (3.21), (3.22) вытекает, что в точке $P$ в направлении вектора $u$

$$
\begin{gathered}
R_{k}^{i}(u)=\bar{R}_{k}^{i}(u)+M\left(\delta_{k}^{i}-\frac{b_{k}+u^{k}}{S} u^{i}\right) \\
+\frac{1}{S}\left(\left\langle F_{i k} u^{i}, \sum_{s=1}^{l} b_{s} F_{s j} u^{j}\right\rangle-\left\langle k(u), \sum_{s=1}^{l} F_{s k} b_{s}\right\rangle\right) u^{i}, \\
\bar{R}_{k}^{i}(u)=\bar{R}_{s k l}^{i} u^{s} u^{l}=\bar{g}^{i j} \bar{R}_{j s k l} u^{s} u^{l} .
\end{gathered}
$$


По формулам Гаусса

$$
\bar{R}_{j s k l}=\sum_{\alpha}\left(\bar{h}_{j k}^{\alpha} \bar{h}_{s l}^{\alpha}-\bar{h}_{j l}^{\alpha} \bar{h}_{s k}^{\alpha}\right)
$$

где $\bar{h}_{i j}^{\alpha}$ - коэффициенты вторых квадратичных форм относительно ортонормированного базиса нормалей $\nu_{\alpha}$.

В точке $P$ коэффициенты вторых квадратичных форм $\bar{h}_{i j}^{\alpha}=f_{i j}^{\alpha}, \bar{g}^{i j}=\delta_{i j}$ и

$$
\bar{R}_{s k l}^{i}=\sum_{\alpha}\left(f_{i k}^{\alpha} f_{s l}^{\alpha}-f_{i l}^{\alpha} f_{s k}^{\alpha}\right)=\left\langle F_{i k}, F_{s l}\right\rangle-\left\langle F_{i l}, F_{s k}\right\rangle
$$

Из (3.23), (3.24), (3.26) следует, что

$$
\bar{R}_{k}^{i}(u)=\left\langle F_{i k}, F_{s l} u^{s} u^{l}\right\rangle-\left\langle F_{i l} u^{l}, F_{s k} u^{s}\right\rangle=\left\langle k(u), F_{i k}\right\rangle-\left\langle F_{i l} u^{l}, F_{s k} u^{s}\right\rangle .
$$

Метрическая форма метрики Рандерса имеет вид (см. [11; п. 1.1, (1.3)])

$$
g_{i j}(x, u)=\frac{S(u)}{\alpha(u)}\left\{\bar{g}_{i j}(x)-\frac{u^{i} u^{j}}{\alpha^{2}(u)}\right\}+\left(c_{i}(x)+\frac{u_{i}}{\alpha(u)}\right)\left(c_{j}(x)+\frac{u_{j}}{\alpha(u)}\right) .
$$

В точке $P$ формула (3.29) принимает вид

$$
g_{i j}(0, u)=S \delta_{i j}-S u^{i} u^{j}+\left(b_{i}+u^{i}\right)\left(b_{j}+u^{j}\right) .
$$

Флаговая кривизна $M^{l}$ в точке $P$ вдоль двумерной плоскости $\sigma$, натянутой на векторы $u$ и $v$, вычисляется по формуле (3.4). Подставим (3.23), (3.27), (3.29) в (3.4). Тогда в точке $P$

$$
\begin{aligned}
g_{u}\left(R_{u}(v), v\right) & =g_{i j}\left(R_{k}^{i}(u) v^{k}\right) v^{j}=\left\{S \delta_{i j}-S u^{i} u^{j}+\left(b_{i}+u^{i}\right)\left(b_{j}+u^{j}\right)\right\} \\
\times & \left\{\bar{R}_{k}^{i}(u) v^{k} v^{j}+M\left(\delta_{k}^{i}-\frac{S_{u_{k}}}{S} u^{i}\right) v^{k} v^{j}\right. \\
+ & \left.\frac{1}{S}\left\{\left\langle F_{m k} u^{m}, \sum_{s} b_{s} F_{s p} u^{p}\right\rangle-\left\langle k(u), \sum_{s} F_{s k} b_{s}\right\rangle\right\} v^{k} v^{j} u^{i}\right\} .
\end{aligned}
$$

Раскроем скобки в уравнении (3.31):

$$
\begin{gathered}
g_{i j}\left(R_{k}^{i}(u) v^{k}\right) v^{j}=\underbrace{S \delta_{i j} \bar{R}_{k}^{i}(u) v^{k} v^{j}}_{(1)}-\underbrace{S \sum_{i, j} \bar{R}_{k}^{i}(u) v^{k} v^{j} u^{i} u^{j}}_{(2)} \\
+\underbrace{\sum_{i, j}\left(b_{i}+u^{i}\right)\left(b_{j}+u^{j}\right) \bar{R}_{k}^{i}(u) v^{k} v^{j}}_{(3)}+\underbrace{S M \delta_{i j}\left\{v^{i}-\frac{\langle b+u, v\rangle}{S} u^{i}\right\} v^{j}}_{(4)} \\
-\underbrace{S M \sum_{i, j} u^{i} u^{j}\left\{v^{i}-\frac{\langle b+u, v\rangle}{F} u^{i}\right\} v^{j}}
\end{gathered}
$$




$$
+\underbrace{M \sum_{i, j}\left(b_{i}+u^{i}\right)\left(b_{j}+u^{j}\right)\left\{v^{i}-\frac{\langle b+u, v\rangle}{S} u^{i}\right\} v^{j}}
$$

(6)

$$
+\underbrace{S \delta_{i j} \frac{1}{S}\left\{\left\langle F_{m k} u^{m}, \sum_{s} b_{s} F_{s p} u^{p}\right\rangle-\left\langle k(u), \sum_{s} b_{s} F_{s k}\right\rangle\right\} u^{i} v^{j} v^{k}}_{(7)}
$$

$$
-\underbrace{\sum_{i, j} S u^{i} u^{j} \frac{1}{S}\left\{\left\langle F_{m k} u^{m}, \sum_{s} b_{s} F_{s p} u^{p}\right\rangle-\left\langle k(u), \sum_{s} b_{s} F_{s k}\right\rangle\right\} u^{i} v^{j} v^{k}}
$$

(8)

$$
+\underbrace{\sum_{i, j}\left(b_{i}+u^{i}\right)\left(b_{j}+u^{j}\right) \frac{1}{S}\left\{\left\langle F_{m k} u^{m}, \sum_{s} b_{s} F_{s p} u^{p}\right\rangle-\left\langle k(u), \sum_{s} b_{s} F_{s k}\right\rangle\right\} u^{i} v^{j} v^{k}} .
$$

(9)

Упростим каждое из слагаемых:

$$
\text { (1) } S \delta_{i j} \bar{R}_{k}^{i}(u) v^{k} v^{j}=S \bar{K}_{\sigma}\left(|u|^{2}|v|^{2}-\langle u, v\rangle^{2}\right)=S \bar{K}_{\sigma}|u \times v|^{2},
$$

где $\bar{K}_{\sigma}-$ секционная кривизна индуцированной римановой метрики $\bar{g}_{i j}$ вдоль площадки $\sigma$.

Подставим значения $\bar{R}_{k}^{i}(u)$ из формулы (3.28) в выражение $(2)$ в $(3.32)$ :

$$
\begin{gathered}
S \sum_{i j} u^{i} u^{j} \bar{R}_{k}^{i}(u) v^{k} v^{j}=S\langle u, v\rangle\left(\left\langle k(u), F_{i k} u^{i} v^{k}\right\rangle-\left\langle F_{i l} u^{i} u^{l}, F_{s k} u^{s} v^{k}\right\rangle\right) \\
=S\langle u, v\rangle\left(\left\langle k(u), F_{i k} u^{i} v^{k}\right\rangle-\left\langle k(u), F_{s k} u^{s} v^{k}\right\rangle\right)=0
\end{gathered}
$$

(3)

$$
\begin{aligned}
& S \sum_{i j}\left(b_{i}+u^{i}\right)\left(b_{j}+u^{j}\right) \bar{R}_{k}^{i}(u) v^{k} v^{j} \\
& \quad=S b_{i} b_{j} \bar{R}_{k}^{i}(u) v^{k} v^{j}+S \sum_{i, j}\left(b_{i} u^{j}+b_{j} u^{i}\right) \bar{R}_{k}^{i}(u) v^{k} v^{j}+S \sum_{i, j} u^{i} u^{j} \bar{R}_{k}^{i}(u) v^{k} v^{j} \\
& \quad=S\langle b+u, v\rangle\left(\left\langle k(u), F_{i k} b^{i} v^{k}\right\rangle-\left\langle F_{i l} b^{i} u^{l}, F_{s k} u^{s} v^{k}\right\rangle\right)
\end{aligned}
$$

(4) $S M \delta_{i j}\left\{v^{i}-\frac{\langle b+u, v\rangle}{S} u^{i}\right\} v^{j}=S M\left(\langle v, v\rangle-\frac{\langle b+u, v\rangle\langle u, v\rangle}{S}\right)$;

(5) $S M \sum u^{i} u^{j}\left\{v^{i}-\frac{\langle b+u, v\rangle}{S} u^{i}\right\} v^{j}=S M\left(\langle u, v\rangle-\frac{\langle b+u, v\rangle\langle u, u\rangle}{S}\right)\langle u, v\rangle$;

(6) $M \sum_{i j}\left(b_{i}+u^{i}\right)\left(b_{j}+u^{j}\right)\left\{v^{i}-\frac{\langle b+u, v\rangle}{S} u^{i}\right\} v^{j}$

$$
=\langle b+u, v\rangle^{2}\left(1-\frac{\langle b+u, u\rangle}{S}\right)=0 .
$$

Флаговый вектор $u$ имеет единичную длину в евклидовой метрике $\langle u, u\rangle=1$ и $S=|u|+\langle b, u\rangle$. 
Запишем выражения для (7)-(9) без сомножителя $\left(\left\langle S_{m k} u^{m} v^{k}, \sum b_{s} S_{s p} u^{p}\right\rangle-\right.$ $\left.\left\langle k(u), \sum b_{s} F_{s k} v^{k}\right\rangle\right):$

(7) $S \delta_{i j} u^{i} v^{j}=S\langle u, v\rangle$;

(8) $S \sum_{i, j} u^{i} u^{j} u^{i} v^{j}=S\langle u, u\rangle\langle u, v\rangle=S\langle u, v\rangle$;

(9) $\frac{1}{S} \sum_{i, j}\left(b_{i}+u^{i}\right)\left(b_{j}+u^{j}\right) u^{i} v^{j}=\frac{1}{S}\langle b+u, u\rangle\langle b+u, v\rangle=S\langle b+u, v\rangle$.

Подставим полученные выражения в (3.31). Получим

$$
\begin{aligned}
g_{u}\left(R_{u}(v), v\right)=\underbrace{S \bar{K}_{\sigma}|u \times v|^{2}}_{(1)}-\underbrace{S\langle b+u, v\rangle\left(\left\langle k(u), F_{i k} b^{i} v^{k}\right\rangle-\left\langle F_{i l} b^{i} u^{l}, F_{s k} u^{s} v^{k}\right\rangle\right)}_{(3)} \\
+\underbrace{S M\left(\langle v, v\rangle-\frac{\langle b+u, v\rangle\langle u, v\rangle}{S}\right)}_{(5)}-\underbrace{S M\left(\langle u, v\rangle-\frac{\langle b+u, v\rangle\langle u, u\rangle}{S}\right)\langle u, v\rangle}_{(5)} \\
+\left(\left\langle F_{m k} u^{m} v^{k}, \sum b_{s} F_{s p} u^{p}\right\rangle-\left\langle k(u), \sum b_{s} F_{s k} v^{k}\right\rangle\right)+S\langle b+u, v\rangle .
\end{aligned}
$$

Упростив, получим следующее выражение:

$$
\begin{aligned}
g_{u}\left(R_{u}(v), v\right) & =S \bar{K}_{\sigma}|u \times v|^{2}+S M(\langle v, v\rangle\langle u, u\rangle-\langle u, v\rangle)^{2} \\
& =S|u \times v|^{2}\left(\bar{K}_{\sigma}+M\right) .
\end{aligned}
$$

Упростим знаменатель $g_{u}\langle u, u\rangle g_{u}\langle v, v\rangle-g_{u}\langle u, v\rangle^{2}$, учитывая, что из однородности метрики следует

$$
g_{u}\langle u, u\rangle=S^{2}
$$

Из формулы (3.29) получим

$$
\begin{aligned}
g_{u}\langle v, v\rangle & =g_{i j} v^{i} v^{j}=S \delta_{i j} v^{i} v^{j}-S \sum_{i, j} u^{i} u^{j} v^{i} v^{j}+\left(b_{i}+u^{i}\right)\left(b_{j}+u^{j}\right) v^{i} v^{j} \\
& =S\langle v, v\rangle-S\langle u, v\rangle^{2}+\langle b+u, v\rangle^{2} .
\end{aligned}
$$

Так как $\langle u, u\rangle=1$, то

$$
\begin{gathered}
g_{u}\langle v, v\rangle=S|u \times v|^{2}+\langle b+u, v\rangle^{2}, \\
g_{u}\langle u, v\rangle=g_{i j} u^{i} v^{j}=S\langle u, v\rangle-S\langle u, u\rangle\langle u, v\rangle+\langle b+u, u\rangle\langle b+u, v\rangle=S\langle b+u, v\rangle .
\end{gathered}
$$

Из (3.44)-(3.46) следует, что

$$
g_{u}\langle u, u\rangle g_{u}\langle v, v\rangle-g_{u}\langle u, v\rangle^{2}=S^{3}|u \times v|^{2} .
$$

Из (3.43), (3.47) получим, что флаговая кривизна $K_{\sigma}(u)$ подмногообразия $M^{l}$ в пространстве Рандерса $R^{n+1}$ в точке $P$ в направлении касательного вектора $u$ и двумерной площадки $\sigma$, натянутой на векторы $u, v$, равна

$$
K_{\sigma}(u)=\frac{\bar{K}_{\sigma}+M}{S^{2}},
$$


где $M=\frac{3 \Phi^{2}}{4 S^{2}}-\frac{\Psi}{2 S}$. Подставив значения $\Phi(P), \Psi(P)$ из $(3.20),(3.21)$, получим

$$
M=\frac{3\langle k(u), b\rangle^{2}}{4 S^{2}}-\frac{\frac{\partial}{\partial s}\langle k(u), b\rangle}{2 S} .
$$

Подставим значения $M$ из (3.49) в (3.48). Тогда

$$
K_{\sigma}(u)=\frac{\bar{K}_{\sigma}}{S^{2}}+\frac{3}{4 S^{4}}\langle k(u), b\rangle^{2}-\frac{1}{2 S^{3}} \frac{\partial}{\partial s}\langle k(u), b\rangle,
$$

где дифференцирование ведется по длине дуги геодезической $\bar{M}^{l} \subset E^{n+1}$, касательной к вектору $u \in T_{P} \bar{M}^{l}$. Из (3.50) следует связь между финслеровой кривизной Риччи $M^{l}$ и римановой кривизной Риччи $\bar{M}^{l}$ :

$$
\operatorname{Ric}(u)=\frac{\overline{\operatorname{Ric}}(u)}{S^{2}}+(l-1)\left(\frac{3}{4 S^{4}}\langle k(u), b\rangle^{2}-\frac{1}{2 S^{3}} \frac{\partial}{\partial s}\langle k(u), b\rangle\right) .
$$

Лемма 2 доказана.

СледствиЕ. Если кривизна Риччи гиперповерхности $M^{l}$ в пространстве Рандерса $\operatorname{Ric}(u) \geqslant 0$, то риманова кривизна Риччи гиперповерхности $\bar{M}^{l}$ в наложенном евклидовом пространстве $\overline{\operatorname{Ric}}(u) \geqslant 0$.

Допустим, это не так. Тогда на гиперповерхности $\bar{M}^{l}$ в точке $P$ есть единичный вектор $u_{0}$ такой, что нормальная кривизна гиперповерхности $\bar{M}^{l}$ в этом направлении равна нулю. Если $u_{0}$ не главное направление, соответствующее нулевому собственному значению, то покажем, что $\overline{\operatorname{Ric}}\left(u_{0}\right)<0$. Выберем координаты в точке $P$, как в лемме 2 . Тогда в этой точке коэффициенты вторых квадратичных форм относительно нормали $\nu$ гиперповерхности $\bar{M}^{l}$ имеют вид $\bar{h}_{i j}=f_{i j}$. По формуле Гаусса секционная кривизна в направлении двумерной площадки $\sigma$, которая проходит через $u_{0}$, равна

$$
\bar{K}_{\sigma}=-\left(\bar{h}_{i j} u_{0}^{i} a^{j}\right)^{2}=-\bar{h}\left(u_{0}, a\right)^{2},
$$

где $a=\left(a^{1}, \ldots, a^{l}\right) \in \sigma-$ единичный ортогональный к $u_{0}$ вектор. Кривизна Риччи

$$
\overline{\operatorname{Ric}}\left(u_{0}\right)=-\sum_{i=1}^{l-1} \bar{h}\left(u_{0}, e_{i}\right)^{2}
$$

где $e_{s}, s=1, \ldots, l-1,-$ ортонормированный набор векторов в касательном пространстве $T_{P} \bar{M}^{l}$, ортогональных вектору $u_{0}$. Если $\bar{h}\left(u_{0}, e_{i}\right)=0$ для всех векторов $e_{i}$, то $u_{0}$ является главным направлением, соответствующим нулевому собственному значению, и $\overline{\operatorname{Ric}}\left(u_{0}\right)=0$. Если это не так, то $\overline{\operatorname{Ric}}\left(u_{0}\right)<0$. Выражение $\frac{\partial}{\partial s}\langle k(u), b\rangle$ может быть неотрицательным или неположительным в зависимости от направления на геодезической, выходящей в направлении $u_{0}$ или $-u_{0}$. Подставив значения $\overline{\operatorname{Ric}}\left(u_{0}\right), k\left(u_{0}\right)$ и $\frac{\partial}{\partial s}\langle k(u), b\rangle$ в формулу $(3.50)$, получим, что $\operatorname{Ric}\left(u_{0}\right)<0$. Это противоречит нашему допущению.

Пусть финслерова кривизна Риччи гиперповерхности $M^{l}$ в пространстве Рандерса $R^{l+1}$ неотрицательна и в каждой точке есть направление $u$ такое, 
что $\operatorname{Ric}(u)=0$. Обозначим $\nu(P)=\operatorname{dim} L(P)$, где $L(P) \subset T_{P} M^{l}-$ линейное подпространство максимальной размерности такое, что для $u \in L(P)$ кривизна Риччи $\operatorname{Ric}(u)=0$, и назовем его нуль-индексом Риччи. Пусть для $P_{0} \in M^{l}$ нуль-индекс Риччи $\nu_{0}=\nu\left(P_{0}\right)=\min _{P \in M^{l}} \nu(P)>0$.

Напомним определение внешнего нуль-индекса.

Внешним нуль-индексом $\bar{\mu}(Q)$ точки $Q \in \bar{M}^{l}$ в евклидовом пространстве $E^{n+1}$ называется максимальная размерность подпространства $\bar{L}(Q)$ касательного пространства $T_{Q} \bar{M}^{l}$ такого, что

$$
\bar{h}_{i j}(\xi) y^{i}=0
$$

для любого $y \in \bar{L}(Q), \bar{h}_{i j}(\xi)$ - вторая квадратичная форма для любой нормали $\xi \in N_{Q} \bar{M}^{l}$.

Лемма 3. В окрестности точки $P_{0}$ распределение $L(P)$ интегрируемо. Слоями являются вполне геодезические плоские подмногообразия $R^{\nu}$ обгемлющего пространства Рандерса. Если гиперповерхность $M^{l}$ полная, то на ней лежит плоскость размерности $\nu_{0}$ объемлющего пространства, если $\nu_{0}<l, u$ размерности $l-1$, если $\nu_{0}=l$.

ДоказАтельство. Пусть $\operatorname{Ric}(u) \geqslant 0$ и $\operatorname{Ric}\left(u_{0}\right)=0$. Тогда из формулы $(3.51)$ следует, что $\overline{\operatorname{Ric}}\left(u_{0}\right)=0$, и вторая квадратичная форма гиперповерхности $\bar{M}^{l}$ в $E^{l+1}$ неотрицательна. Пусть вектор $u_{0}$ не принадлежит подпространству, отвечающему нулевой главной кривизне гиперповерхности $\bar{M}^{l}$ в евклидовом пространстве. Допустим, что найдется еще вектор $v_{0} \in T_{P} \bar{M}^{l}$, также не принадлежащий нулевому подпространству. Тогда в силу теоремы Гаусса $\overline{\operatorname{Ric}}\left(u_{0}\right)>0$, что противоречит ранее доказанному. Поэтому в этом случае $\bar{\mu}=l-1, \nu_{0}=l$. Если $\nu_{0}<l$, то из предыдущих рассуждений следует $\bar{\mu}=\nu_{0}$.

Если была полна гиперповерхность $M^{l} \subset R^{l+1}$, то полна и гиперповерхность $\bar{M}^{l}$ в евклидовом пространстве $E^{l+1}$. Для подмногообразий в евклидовом пространстве справедлива лемма 3. Так как в силу неотрицательности кривизны $\operatorname{Ric}(u) \mu=\bar{\mu}$, то лемма справедлива и для пространства Рандерса.

ЛЕмма 4 (см. [7]). Пусть $\bar{M}^{l}$ есть регулярное класса $C^{3}$ подмногообразие в евклидовом пространстве $E^{n}$. Предположим, что в окрестности $Q_{0} \in \bar{M}^{l}$ нуль-индекс $\bar{\mu}(Q)=k$ постоянен. Тогда нуль-распределение $\bar{L}(Q)$ интегрируемо и слои являются областями на плоскостях $E^{k}$ обгемлющего пространства. Касательное пространство стационарно вдоль слоя. Если $\bar{M}^{l}-$ полное подмногообразие и $\bar{\mu}\left(Q_{0}\right)=\min _{Q \in \bar{M}^{l}} \bar{\mu}(Q)=k$, то слой, проходящий через точку $Q_{0}$, является плоскостью $E^{k}$.

Х. Рунд ввел определение второй квадратичной формы для гиперповерхностей пространства Минковского (см. [12]), Х. Элиопоулос обобщил его на случай подмногообразий произвольной коразмерности в пространстве Минковского (см. [13]).

Для гиперповерхности в пространстве Минковского $M^{n}$ с индикатрисой $S\left(y^{1}, \ldots, y^{n+1}\right)=1$ и параметрическим заданием гиперповерхности

$$
y^{a}=y^{a}\left(u^{1}, \ldots, u^{n}\right)
$$


нормаль $\nu$ удовлетворяет уравнению (см. [12; гл. V, $§,(7.1)]$ )

$$
\frac{\partial y^{a}}{\partial u^{i}} \nu^{a}=0, \quad \nu^{a}=g^{a}(x, \nu) \nu_{b}, \quad S(\nu)=1, \quad g_{a b}=\frac{1}{2} \frac{\partial^{2} S^{2}}{\partial y^{a} \partial y^{b}} .
$$

Если $M^{n}$ - гиперповерхность, то нормаль в точке $P$ гиперповерхности $M^{n}$ будет радиусом-вектором индикатрисы в той точке, в которой касательная гиперплоскость параллельна касательной гиперплоскости гиперповерхности $M^{n}$ в точке $P$.

Для подмногообразий произвольной коразмерности ковариантные компоненты нормалей удовлетворяют той же системе уравнений.

Для гиперповерхностей компоненты второй квадратичной формы определяются уравнением (см. [12; гл. V, $\S 7,(7.27)])$

$$
h_{i j}=n_{a} I_{i j}^{a} \text {, }
$$

где

$$
\begin{gathered}
I_{i j}^{a}=\frac{\partial y^{a}}{\partial u^{i} \partial u^{j}}-\frac{\partial y^{a}}{\partial u^{s}} \Gamma_{i j}^{* s}+\Gamma_{c d}^{* a} \frac{\partial y^{c}}{\partial u^{i}} \frac{\partial y^{d}}{\partial u^{j}}, \\
\Gamma_{c d}^{* a}=\Gamma_{c d}^{a}-C_{i j}^{k} \Gamma_{i j}^{h} y^{j} \\
\Gamma_{i j}^{h}=g^{h s} \Gamma_{i j, s}, \\
\Gamma_{i j, h}=\frac{1}{2}\left(\frac{\partial g_{i h}}{\partial u^{j}}+\frac{\partial g_{j h}}{\partial u^{i}}-\frac{\partial g_{i j}}{\partial u^{h}}\right), \\
C_{i j}^{k}=g^{k s} C_{i j, s}, \\
C_{i j, s}=\frac{1}{2}\left(\frac{\partial g_{i s}}{\partial u^{j}}+\frac{\partial g_{j s}}{\partial u^{i}}-\frac{\partial g_{i j}}{\partial u^{s}}\right),
\end{gathered}
$$

где $g_{i j}(v)$ - финслерова метрическая форма, индуцированная на гиперповерхности (см. [12]).

Так как для объемлющего пространства Минковского $\Gamma_{c d}^{a}=0$, то коэффициенты второй квадратичной формы принимают следующий вид:

$$
h_{i j}=\nu_{a} \frac{\partial y^{a}}{\partial u^{i} \partial u^{j}} .
$$

Этой же формулой выражаются и коэффициенты вторых квадратичных форм для подмногообразий произвольной коразмерности (см. [13]).

Для подмногообразия $M^{l}$ в пространстве Минковского $M^{n+1}$ рассмотрим наложенное евклидово пространство $E^{n+1}$. На подмногообразии $\bar{M}^{l}$ в $E^{n+1}$, которое как множество точек совпадает с $M^{l}$, индуцируется риманова метрика. Линейные пространства вторых квадратичных форм в той же точке $P \in M^{l}$, $P \in \bar{M}^{l}$ совпадают в силу формулы (3.59) и формулы для вычисления вторых квадратичных форм в евклидовом пространстве.

Введем понятие внешнего нуль-индекса $\mu(Q)$ для подмногообразий пространства Минковского аналогично определению в евклидовом пространстве. Это было сделано в работе [14]. Нуль-индексы $\mu(Q)$ и $\bar{\mu}(Q)$ совпадают. Поэтому в пространстве Минковского справедливо утверждение леммы 4. Только слоями являются плоскости пространства Минковского. 
Для вторых квадратных форм подмногообразия в римановом пространстве введены определения ранга, типа точки, внешнего нуль-индекса (см. [7], [15]). Эти же определения вводятся для подмногообразий в пространстве Минковского.

Пусть $r(Q, \xi)$ есть ранг второй квадратичной формы подмногообразия $M^{l}$ в пространстве Минковского $M^{n+1}$ в точке $Q$ относительно нормали $\xi$.

Рангом второй квадратичной формы поверхности $M^{l}$ в точке $Q$ называется целое неотрицательное число

$$
r(Q)=\max _{\xi \in N_{Q}} r(Q, \xi),
$$

где $N_{Q}$ - нормальное подпространство в точке $Q$.

Пусть вторая квадратичная форма после приведения к диагональному виду имеет $k_{1}$ положительных и $k_{2}$ отрицательных членов. Тогда

$$
j(Q)=\min \left(k_{1}, k_{2}\right) .
$$

Типом точки $Q$ называется число

$$
j(Q)=\min j(Q, \xi),
$$

где минимум берется по всем нормам в точке $Q$, для которых $r(Q, \xi)=r(Q)$.

Если $j(Q)=0$, то существует нормаль в точке, относительно которой вторая квадратичная форма является неотрицательно определенной. При этом если ранг $r(Q)=l$, то относительно некоторой нормали вторая квадратичная форма положительно определена. Если $r(Q)=l-k$, то относительно некоторой нормали ограничение второй квадратичной формы на некоторую $(l-k)$-плоскость будет положительно определенно.

Поверхности с нулевым типом являются в некотором смысле обобщением выпуклых гиперповерхностей на подмногообразия произвольной коразмерности.

ДокАЗАТЕЛЬСТво ТЕОРемы 2. Так как для подмногообразия $M^{l}$ в пространстве Минковского и подмногообразия $\bar{M}^{l}$ в евклидовом пространстве $\mu(Q)=\bar{\mu}(Q), j(Q)=\bar{j}(Q)$, то по теореме 1 подмногообразие $\bar{M}^{l}$ является цилиндром с $k$-мерной образующей, а значит, и подмногообразие $M^{l}$.

Но так как плоскости евклидова пространства являются вполне геодезическими плоскостями пространства Минковского, то теорема доказана.

ДоКАЗАТЕЛЬСТВо ТЕОРЕМЫ 3. Из следствия получается, что наложенная поверхность $\bar{M}^{l}$ в евклидовом пространстве $E^{l+1}$ имеет также неотрицательную кривизну Риччи, а прямая, которая принадлежит $M^{l}$, является прямой и евклидова пространства, а гиперповерхность $\bar{M}^{l}$ является полной. Поэтому по теореме Чигера-Громолла (см. [3]) гиперповерхность $\bar{M}^{l}$ с точки зрения внутренней геометрии является метрическим произведением. Но имеет место

Лемма 5 (см. [7], [16]). Пусть подмногообразие $\bar{M}^{l}$ в $E^{n}$ является метрическим произведением $\bar{M}^{l}=M^{l-1} \times E^{1}$ и образ $\left(P \times E^{1}\right)$, где $P$ - фиксированная точка $M^{l}$, является прямой $\gamma$ обгемлющего евклидова пространства. Тогда $\bar{M}^{l}$ является иилиндром с образующей прямой $\gamma$. 
Так как прямые наложенного евклидова пространства являются и прямыми пространства Рандерса и при этом параллельность сохраняется, то гиперповерхность $M^{l}$ является цилиндром.

ДокАЗАТЕЛЬСтво теоремы 4. Так как аналог леммы 4 имеет место в пространстве Минковского, то на гиперповерхности $M^{l}$ лежит $k$-мерная плоскость $R^{k}$. Тогда, применяя теорему 3, мы получим нужное утверждение.

ДоКАЗАТЕЛЬСТво тЕОРЕмы 5. Она следует из леммы 3 и теоремы 4.

ДокАЗАтельство теоремы 6. Из (3.51) следует, что кривизна Риччи подмногообразия $\bar{M}^{l}$ в евклидовом пространстве $E^{n}$ неотрицательная. Дальнейшее доказательство совпадает с доказательством теоремы 3. Если подмногообразие $\bar{M}^{l}$ лежит в гиперплоскости, перпендикулярной вектору $b$, то утверждение теоремы верно при условии неотрицательности финслеровой кривизны Риччи $M^{l}$.

ДОКАЗАТЕЛЬСТВо ТЕОРЕМЫ 7. Из условия теоремы и обобщения леммы 4 на случай пространства Минковского следует, что на $M^{l}$ лежит плоскость $R^{\nu_{0}}$ пространства Рандерса. Из условия на кривизну Риччи следует, что поверхность $\bar{M}^{l}$ имеет неотрицательную кривизну Риччи. Дальнейшее доказательство совпадает с доказательством теоремы 4.

\section{§ 4. Положительность флаговой кривизны гиперповерхностей в пространстве Рандерса}

Из выпуклости гиперповерхности в пространстве Рандерса не следует неотрицательность ее флаговой кривизны. Однако при некоторых ограничениях на геометрию гиперповерхности в наложенном евклидовом пространстве такая зависимость имеет место.

Теорема 8. Пусть $M^{l}$ есть $C^{3}$-гладкая гиперповерхность в пространстве Рандерса $R^{l+1}$. Пусть $\bar{M}^{l}$ в наложенном евклидовом пространстве имеет положительную секционную кривизну $\bar{K}_{E}$, нормальные кривизны наложенной гиперповерхности $\bar{M}^{l}$ удовлетворяют неравенству $0<k_{1} \leqslant k_{n} \leqslant k_{2} u$ $|b|<\frac{2 k_{1} / k_{2}}{2 k_{1} / k_{2}+1}$.

Если вдоль каждой геодезической на гиперповерхности $\bar{M}^{l}$ для нормалъной кривизны выполняется неравенство

$$
\left|\frac{\partial k}{\partial s}\right|<2 \bar{K}_{E} \sqrt{3\left(\frac{k_{1}}{k_{2}}-\frac{|b|}{2(1-|b|)}\right)},
$$

то флаговая кривизна $K_{R}$ положительна.

ДокАЗАТЕЛЬСтво. В некоторой окрестности произвольной точки $P \in \bar{M}^{l} \subset$ $E^{l+1}$ гиперповерхность задается явно:

$$
z=f\left(x^{1}, \ldots, x^{l}\right), \quad f(0)=0, \quad f_{i}(P)=0 .
$$


Для касательного направления $\tau \in T_{P} M^{l}$ рассмотрим нормальную геодезическую $\gamma, \gamma(0)=P, \dot{\gamma}(0)=\tau$. Вектор главной нормали геодезической имеет направление оси $z$. Тогда формула (3.50) принимает вид

$$
K_{R}(\tau)=\frac{\bar{K}_{E}}{S^{2}}+\frac{3}{4} \frac{b_{l+1}^{2} k^{2}(\tau)}{S^{4}(\tau)}-\frac{1}{2 S^{3}(\tau)} \frac{\partial}{\partial s}(k\langle b, n\rangle) .
$$

Действительно,

$$
k(\tau)=\frac{f_{i j} \frac{d x^{i}}{d s} \frac{\partial x^{j}}{\partial s}}{\left(\delta_{i j}+f_{i} f_{j}\right) \frac{d x^{i}}{d s} \frac{d x^{j}}{d s}},
$$

где $x^{i}=x^{i}(s)$ - параметрическое уравнение геодезической на $\bar{M}^{l}$. Тогда в точке $P$, учитывая, что $\frac{d^{2} x^{i}}{d s^{2}}=0, f_{i}(0)=0$, получаем

$$
\left.\frac{\partial\langle b, \nu\rangle}{\partial s}\right|_{P}=-f_{i j} \frac{d x^{i}}{d s} b^{j} .
$$

В точке $P$ формула (3.50) примет вид

$$
K_{R}(\tau)=\frac{\bar{K}_{E}}{S^{2}(\tau)}+\frac{3}{4} \frac{b_{l+1}^{2} k^{2}(\tau)}{S^{4}(\tau)}-\frac{1}{2} \frac{\frac{\partial k}{\partial s} b_{l+1}}{S^{3}(\tau)}+\frac{1}{2} \frac{k(\tau) f_{i j} b^{j} \frac{d x^{i}}{d s}}{S^{3}(\tau)}
$$

Так как нормальные кривизны гиперповерхности $\bar{M}^{l}$ удовлетворяют неравенству $0<k_{1} \leqslant k \leqslant k_{2}$, то

$$
-k_{2}|b| \leqslant f_{i j} b^{j} \frac{d x^{i}}{d s} \leqslant k_{2}|b| .
$$

Заметим, что $F(\tau)=1+\langle b, \tau\rangle$. Поэтому

$$
0<1-|b| \leqslant F(\tau) \leqslant 1+|b| .
$$

Формула для флаговой кривизны есть квадратный трехчлен от $b_{l+1}$. Достаточным условием положительности флаговой кривизны является условие на отрицательность дискриминанта

$$
\left(\frac{d k(\tau)}{d s^{2}}\right)^{2}<12 k^{2}(\tau)\left(\bar{K}_{E}+\left(f_{i j} b^{j} \frac{d x^{i}}{d s}\right) k(\tau)\right) .
$$

Это неравенство эквивалентно системе неравенств

$$
\begin{gathered}
\left(\frac{d k(\tau)}{d s}\right)<2 k(\tau) \sqrt{3\left(\bar{K}_{E}+\frac{\left(f_{i j} b^{j} \frac{d x^{i}}{d s}\right) k(\tau)}{2 F(\tau)}\right)}, \\
\bar{K}_{E}+\frac{f_{i j} b^{j} \frac{d x^{i}}{d s}}{2 F(\tau)}>0 .
\end{gathered}
$$


Используя оценки (4.1), (4.2), мы получим, что неравенства

$$
\begin{gathered}
\frac{|b|}{2(1-|b|)}<\frac{k_{1}}{k_{2}} \\
\left|\frac{d k(\tau)}{d s}\right|<2 \bar{K}_{E} \sqrt{3\left(\frac{k_{1}}{k_{2}}-\frac{|b|}{2(1-|b|)}\right)}
\end{gathered}
$$

обеспечат выполнение неравенств предыдущей системы, и при этих условиях флаговая кривизна гиперповерхности в пространстве Рандерса будет положительной.

Для двумерных поверхностей в трехмерном пространстве Рандерса это было доказано в [17].

Искренне благодарю К. Д. Драча за внимательное прочтение рукописи, а И. В. Шпоту - за подготовку рукописи к печати.

\section{Список литературы}

[1] S. Cohn-Vossen, "Totalkrümmung und geodätische Linien auf einfachzusammenhängenden offenen vollständigen Flächenstücken", Матем. сб., 1(43):2 (1936), 139-164.

[2] В. А. Топоногов, "Метрическое строение римановых пространств неотрицательной кривизны, содержащих прямые линии", Сиб. матем. журн., 5:6 (1964), 1358-1369.

[3] J. Cheeger, D. Gromoll, "The splitting theorem for manifolds of nonnegative Ricci curvature", J. Differential Geometry, 6 (1971), 119-128.

[4] J.-H. Eschenburg, "The splitting theorem for space-times with strong energy condition", J. Differential Geom., 27:3 (1988), 477-491.

[5] G. J. Galloway, "The Lorentzian splitting theorem without the completeness assumption", J. Differential Geom., 29:2 (1989), 373-387.

[6] Shin-ich Ohta, Splitting theorem for Finsler manifolds of nonnegative Ricci curvature, arXiv: $1203.0079 \mathrm{v} 2$.

[7] А.А. Борисенко, "Внешняя геометрия сильно параболических многомерных подмногообразий”, УМH, 52:6(318) (1997), 3-52; англ. пер.: А. А. Borisenko, "Extrinsic geometry of strongly parabolic multidimensional submanifolds", Russian Math. Surveys, 52:6 (1997), 1141-1190.

[8] P. Hartman, "On the isometric immersions in Euclidean space of manifolds with nonnegative sectional curvatures. II", Trans. Amer. Math. Soc., 47:2 (1970), 529-540.

[9] P. Hartman, L. Nirenberg, "On spherical image maps whose Jacobians do not change sign", Amer. J. Math., 81:4 (1959), 901-920.

[10] R. Sacksteder, "On hypersurfaces with no negative sectional curvatures", Amer. J. Math., 82:3 (1960), 609-630.

[11] Shiing-Shen Chern, Zhongmin Shen, Riemann-Finsler geometry, Nankai Tracts Math., 6, World Sci. Publ., Hackensack, NJ, 2005, x+192 pp.

[12] Х. Рунд, Дифференииальная геометрия финслеровых пространств, Наука, М., 1981, 504 с.; пер. с англ.: Н. Rund, The differential geometry of Finsler spaces, Grundlehren Math. Wiss., 101, Springer-Verlag, Berlin-Göttingen-Heidelberg, 1959, xiii+284 pp.

[13] H. A. Eliopoulos, "Subspaces of a generalized metric space", Canad J. Math., 11 (1959), 235-255. 
[14] А.А. Борисенко, Строение поверхностей с вырожденным сферическим изображением, Дисс. ... канд. физ.-матем. наук, Харьковский гос. ун-т, Х., 1973.

[15] А.А. Борисенко, "О строении 1-мерных поверхностей с вырожденной второй квадратичной формой в $n$-мерном евклидовом пространстве", Укр. геом. сб., 1973, № 13, 18-27.

[16] А. А. Борисенко, "О строении непрерывной поверхности, содержащей прямую", Укр. геом. сб., 1973, № 14, 21-24.

[17] А. А. Борисенко, С. В. Мирошниченко, "О флаговой кривизне двумерных поверхностей в трехмерном пространстве Рандерса", Докл. НАН Украинъ, 2012, № 11, $7-14$.

Александр Андреевич Борисенко (Alexander A. Borisenko)

Сумский государственный университет, Украина

E-mail: aborisenk@gmail.com
Поступила в редакцию 14.01.2014 и 01.04.2014 